\title{
Perfil dos laboratórios de citopatologia do Sistema Único de Saúde
}

\section{Assessment of cytology laboratory performance within the Brazilian Unified Health System}

Luiz Claudio Santos Thuler'; Lucilia Maria Zardo²; Luiz Carlos Zeferino

\begin{abstract}
unitermos resumo
Exame colpocitológico

Objetivo: Conhecer o perfil dos laboratórios de citopatologia que prestam serviços ao Sistema Único de

Qualidade

Saúde (SUS) e elaborar indicadores de qualidade dos exames citopatológicos. Métodos: Foi realizada uma enquete postal dirigida aos 1.028 laboratórios que prestaram serviço ao SUS e participaram do Sistema

Padrōes de referência de Informação do Câncer do Colo do Útero (SISCOLO) em 2002. As informações referentes aos exames

Estatistica citopatológicos constantes do SISCOLO e disponibilizadas na página do Departamento de Informática do Sistema Único de Saúde (DATASUS) na Internet (www.datasus.gov.br) para o mesmo ano foram analisadas. Resultados: Dos 1.028 laboratórios que informaram os resultados dos exames citopatológicos cervicovaginais ao DATASUS, em 2002, 739 responderam à enquete (71,9\%). Desses, 18,9\% processaram 15 mil exames ou mais no ano; $70,2 \%$ eram privados; $50,2 \%$ encontravam-se vinculados à gestão municipal; e $48,7 \%$ realizavam exames histopatológicos. Em $74,8 \%$ dos casos o responsável técnico pelo laboratório era médico. Dos 10.505.773 exames incluídos no SISCOLO em 2002, uma parcela das amostras $(1,66 \%)$ foi considerada insatisfatória. As alterações detectadas foram: células escamosas atípicas de significado indeterminado (ASCUS), 1,26\%; papilomavírus humano (HPV), 0,93\%; neoplasia intra-epitelial cervical I (NIC I), 0,84\%; NIC II, 0,20\%; NIC III, 0,17\%; carcinoma escamoso invasivo, 0,05\%; células glandulares atípicas de significado indeterminado (AGUS), 0,14\%; adenocarcinoma in situ, 0,01; e adenocarcinoma invasivo, 0,01\%. Conclusão: Este estudo permitiu avaliar o perfil dos laboratórios de citopatologia que prestaram serviço ao SUS em 2002, e o SISCOLO se mostrou um excelente instrumento para o monitoramento da qualidade dos exames citopatológicos realizados no país.
\end{abstract}

\section{abstract}

Objective: To assess the profile of cytopathology laboratories which render services to the Brazilian Unified Health System (SUS), and to create quality indicators for cytopathological exams. Methods: A postal survey of 1,028 laboratories that render services to the SUS and participated in the Cervix Cancer Information System (SISCOLO) in the year 2002. Information concerning cytopathological exams from the SISCOLO, available on the Internet (www.datasus.gov.br) for the same year was analyzed. Results: Out of the 1,028 laboratories which reported results of cervix-vaginal cytopathology exams to DATASUS in 2002, 739 answered the survey (71.9\%). From these, $18.9 \%$ processed 15,000 exams or more in that year; $70.2 \%$ were private; $50.2 \%$ were vinculated to municipal administrations; and $48.7 \%$ performed histopathological exams. In $74.8 \%$ of cases, a physician was in charge of the technical responsibility. Out of 10,505.773 exams included in the SISCOLO in 2002, 1.66\% of samples were considered unsatisfactory. The following alterations were detected: atypical squamous cells of undetermined significance (ASCUS), 1.26\%; human papillomavirus (HPV), 0.93\%; cervical intraepithelial neoplasia I (CIN I), 0.84\%, CIN II =0.20\%, CIN III =0.17\%; invasive squamous carcinoma, $0.05 \%$, atypical glandular cells of undetermined significance (AGUS), $0.14 \%$, in situ adenocarcinoma, 0.01; and invasive adenocarcinoma, $0.01 \%$. Conclusion: This study made it possible to assess the profile of cytopathology laboratories, which rendered services to the SUS in 2002, and the SISCOLO proved to be an excellent tool to assess the quality of cytopathological exams performed in Brazil. key words

Pap smears

Quality

Reference standards

Statistics

1. Médico epidemiologista do Instituto Nacional de Câncer (INCA); professor-adjunto da Universidade Federal do Estado do Rio de Janeiro (UNIRIO).

2. Médica citopatologista chefe do Serviço Integrado Tecnológico em Citopatologia do Instituto Nacional de Câncer (SITEC/INCA).

3. Médico ginecologista e professor associado da Faculdade de Ciências Médicas da Universidade Estadual de Campinas (FCM/UNICAMP).

Trabalho realizado no INCA. 


\section{Introdução}

O rastreamento do câncer do colo do útero por meio da citologia cervicovaginal tem sido reconhecido como o método de rastreamento mais bem-sucedido na história da medicina. Embora as alterações citopatológicas tenham sido descritas inicialmente em 1928, por Papanicolaou ${ }^{(22)} \mathrm{e}$ Babes $^{(5)}$, no Brasil só há referência à utilização da citologia no diagnóstico do câncer em 1942, na tese de docência de Antonio Vespasiano Ramos, intitulada "Novo método de diagnóstico precoce do câncer uterino". No entanto, o primeiro serviço de citopatologia só foi fundado em 1956, na Santa Casa de Belo Horizonte ${ }^{(28)}$. Desde então, o número de laboratórios que realizam exames citopatológicos no país vem crescendo progressivamente. Um registro do Instituto Nacional de Câncer (INCA), para 1989, indicava a existência de 361 laboratórios no país(1). Para o período com dados disponibilizados pelo Departamento de Informática do Sistema Único de Saúde (DATASUS), a partir do Sistema de Informação do Câncer do Colo do Útero (SISCOLO), observa-se que houve aumento de $83 \%$ no número de laboratórios de citopatologia com informações disponíveis, passando de 589, em 2000, para 1.075, em 2004(8). Paralelamente, as estimativas do INCA apontam para um crescente percentual da população feminina brasileira submetida a exames citopatológicos do colo do útero, com coberturas de $1,2 \%$, em $1984^{(4)} ; 7,7 \%$, em $1987^{(1)} ; 64 \%$, em $1994^{(18)}$ e $82 \%$ (valor mediano), em 2002-2003 ${ }^{(12)}$.

Contudo, a efetividade do exame de Papanicolaou depende de uma seqüência de eventos, que vão desde o convencimento da mulher a submeter-se ao "exame preventivo" até a adequada abordagem das lesões eventualmente identificadas ${ }^{(25)}$. Ressalte-se que todas as etapas envolvidas na captação das mulheres, como coleta do exame citológico, transporte e processamento das lâminas, identificação de lesões e, finalmente, entrega dos resultados, tratamento e seguimento das mulheres com alterações, devem acontecer em cadeia, de forma sincronizada e com a máxima qualidade. Qualquer falha em uma dessas etapas pode comprometer o impacto do rastreamento da saúde da população.

Nesse cenário, assumem importância capital as atividades de controle e garantia da qualidade do exame citológico, estabelecendo que para um acompanhamento de excelência é necessário o monitoramento sistemático das atividades desenvolvidas, desde a coleta do esfregaço cervicovaginal até a entrega do resultado ao médico. Segundo a American Society of Cytopathology (ASC) ${ }^{(3)}$ podem ser utilizadas diferentes estratégias para o controle e a garantia da qualidade do exame citológico, entre as quais destacamos:

- a avaliação pré-analítica;

- revisão de casos;

- correlações cito-histológica e clínica;

- avaliação do desempenho do exame;

- regulamentação do exercício da profissão;

- educação médica continuada;

- teste de proficiência.

Por sua vez, a Organización Panamericana de la Salud $(\mathrm{OPS})^{(21)}$ propõe que o monitoramento dos laboratórios de citopatologia deva ser baseado em três indicadores: produtividade, qualidade e desempenho. O primeiro é obtido pela divisão do número de exames lidos pelo número de citotecnólogos; já a qualidade é medida pela proporção de informes por categoria diagnóstica, enquanto o desempenho é avaliado pelo grau de concordância cito-histológica. Além disso, a OPS considera como critérios indispensáveis para participar de sua Red Panamericana de Citologia (REDPAC) contar com um médico citopatologista e processar pelo menos 15 mil exames/ano. Outro indicador considerado pela OPS é ter estreita vinculação com laboratórios de anatomia patológica.

Entre os indicadores propostos para avaliar a qualidade da citologia cervicovaginal, destacam-se a distribuição percentual de diagnósticos selecionados, a taxa de exames insatisfatórios e a razão entre o número de diagnósticos de células escamosas atípicas de significado indeterminado (ASCUS) e o número de lesões intra-epiteliais escamosas de alto grau ${ }^{(17,26)}$.

O conhecimento do perfil dos laboratórios de citopatologia prestadores de serviço ao Sistema Único de Saúde (SUS) e da qualidade dos resultados dos exames neles processados é etapa fundamental na consolidação das estratégias de rastreamento do câncer do colo do útero no país. No Brasil, esse conhecimento atualmente é fragmentado, pontual e incompleto. Portanto, visando contribuir para ampliar esse conhecimento, realizamos esta pesquisa que tem por objetivos principais conhecer o perfil dos laboratórios de citopatologia que prestam serviços ao SUS e analisar alguns indicadores para avaliação da qualidade dos exames citopatológicos.

\section{Metodologia}

Para obter dados referentes ao perfil dos laboratórios de citopatologia, solicitamos ao DATASUS a listagem completa 
com os endereços das unidades que realizaram exame citopatológico cervicovaginal (código 12.011.01) para o SUS em 2002. Um questionário de uma página, cujo preenchimento exigia no máximo 10 minutos, foi enviado aos 1.028 laboratórios identificados. Uma vez que o estado do Pará não prestava informações ao DATASUS em 2002, nenhuma unidade desse estado foi convidada a participar da enquete. Foi estabelecido que a participação seria voluntária e que as respostas teriam garantia de sigilo. Nesse questionário foram solicitados dados referentes à natureza da organização (se privada ou pública), à forma de gestão (se municipal, estadual ou federal) e ao tipo de unidade (se laboratório isolado ou pertencente a uma unidade hospitalar e/ou ambulatorial). Também foi pesquisado se eles realizavam exames histopatológicos e sobre a categoria profissional do responsável técnico e dos funcionários do laboratório.

O número de exames citopatológicos realizados no SUS foi estimado a partir do Sistema de Informações Ambulatoriais do Sistema Único de Saúde (SIA/SUS), tomando-se o "número de exames apresentados" ao invés do "número de exames pagos", por aproximar-se mais do número real de exames processados, conforme orientação do INCA ${ }^{(13)}$.

As informações referentes aos exames citopatológicos foram extraídas do SISCOLO, a partir da página do DATASUS na internet (www.datasus.gov.br). Visto que os dados do estado do Pará foram recentemente disponibilizados, o número total de laboratórios com informações sobre os exames citopatológicos ampliou-se para 1.088 (1.028 laboratórios com informações disponíveis em $2002+60$ laboratórios do estado do Pará cujas informações foram encaminhadas ao DATASUS em data posterior).

A terminologia empregada para a emissão dos laudos dos exames citológicos, em 2002, correspondia àquela preconizada pelo Ministério da Saúde (MS) e pela Sociedade Brasileira de Citopatologia (SBC) ${ }^{(14)}$, desde 1993, e implantada nacionalmente a partir de 1998. Essa nomenclatura adota a classificação de Richart para as lesões pré-invasoras associada à conceituação de displasias e carcinoma in situ, assim como de alguns diagnósticos citológicos introduzidos pelo sistema de Bethesda. As alterações de células epiteliais escamosas foram classificadas em ASCUS, efeito citopático compatível com papilomavírus humano (HPV), neoplasia intra-epitelial cervical (NIC) graus I (displasia leve), II (displasia moderada) e III (displasia acentuada; carcinoma in situ) e carcinoma invasivo de células escamosas, enquanto as alterações em células epiteliais glandulares foram classificadas como células glandulares atípicas de significado indeterminado (AGUS), adenocarcinoma in situ e adenocarcinoma invasivo ${ }^{(14)}$. Essa nomenclatura foi mantida neste estudo, pois era oficialmente utilizada no SUS no período pesquisado.

As informações foram armazenadas eletronicamente no programa Excel (Microsoft Corporation, versão 5.1, 2002) e analisadas com o apoio do programa Epilnfo (versão 3.3, 2004). Os dados referentes às características dos laboratórios, qualidade da amostra e alterações detectadas foram apresentados como números e porcentagens para cada unidade da Federação (UF). Conforme recomendação do College of American Pathologists (CAP) ${ }^{(6)}$, foram calculados os percentis 5, 10, 25, 50, 75, 90 e 95 para as porcentagens das seguintes alterações: ASCUS; lesão de baixo grau (HPV e NIC I); lesão de alto grau (NIC II e III); razão ASCUS/alterações em células escamosas (ACE): ACE = HPV + NIC I + NIC II + NIC III + carcinoma escamoso invasor); AGUS e amostra insatisfatória. Outros indicadores utilizados foram presença de médico patologista e/ou citopatologista como responsável técnico e na equipe; quantidade de exames/ano 15 mil e realização de exames histopatológicos, conforme proposta da OPS(21).

Com o objetivo de comparar os laboratórios participantes e aqueles que não responderam à enquete, os testes $t$ de Student e da mediana ${ }^{(17)}$ foram usados para comparar, respectivamente, a média e a mediana de algumas variáveis disponíveis. Para identificação de fatores associados a uma freqüência elevada de ASCUS, AGUS, razão ASCUS/ACE e amostras insatisfatórias foram calculados testes do quiquadrado $\left(\chi^{2}\right)$. Os valores críticos usados para estabelecer os pontos de corte em cada um desses desfechos deveriam ser superiores ao percentil 75. Valores de $p<0,05$ foram considerados estatisticamente significativos.

\section{Resultados}

\section{Descrição das características dos laboratórios ( $n$ = 739 laboratórios)}

Foram enviados questionários a 1.028 laboratórios que realizaram exames citopatológicos cervicovaginais para o SUS no ano de 2002. Depois de excluídas as respostas duplas, 739 laboratórios foram incluídos no estudo, o que equivale a uma taxa de respostas de $71,9 \%$. A fim de identificar se os laboratórios participantes diferiam daqueles que não responderam à enquete $(n=349)$, procedeu-se à comparação dos percentuais medianos de resultados insatisfatórios, ASCUS e AGUS observados nos dois grupos. Os 
resultados sugeriram não haver diferença estatisticamente significativa entre laboratórios participantes e não-participantes para essas variáveis $(p>0,05)$. Também não houve diferença estatisticamente significativa no número médio de exames (10.306 versus 8.279 , respectivamente; $p>0,05$ ) realizado pelos dois grupos, em 2002 (dados não mostrados).

Conforme se observa na Tabela 1, com a inclusão dos dados do estado do Pará, os 739 laboratórios respondentes equivalem a $67,9 \%$ do total de laboratórios prestadores de serviços ao SUS em 2002. Desses, somente $18,9 \%$ processaram 15 mil exames ou mais pelo SUS, enquanto $70,2 \%$ eram privados, $25,1 \%$ eram públicos e/ou sem fins lucrativos (fundações beneficentes ou organizações não-governamentais [ONGs]) e não foi informada a natureza da organização nos demais $4,7 \%$. Por outro lado, mais da metade $(50,2 \%)$ das unidades encontrava-se vinculada à gestão municipal, enquanto em $24 \%$ dos casos o tipo de gestão era estadual. Essa informação não foi fornecida por $25,8 \%$ dos laboratórios. A partir dos dados da Tabela 1 observou-se ainda que a maioria dos laboratórios prestadores de serviço ao SUS funcionava de forma isolada $(60,1 \%)$, não estando vinculada a nenhuma unidade de saúde. A realização do exame histopatológico foi referida por $48,7 \%$ dos laboratórios.

Quanto à categoria profissional do responsável técnico pelo laboratório, observou-se que 553 laboratórios $(74,8 \%)$ eram chefiados por médicos, dos quais 507 por anatomopatologistas e/ou citopatologistas. No que se refere aos profissionais de nível superior envolvidos na realização dos exames citopatológicos do colo do útero no SUS, $60,2 \%$ dos laboratórios referiram a presença de médicos citopatologistas, enquanto quase metade deles $(48,6 \%)$ possuía em seus quadros médicos anatomopatologistas e 13,8\%, médicos patologistas clínicos. Outros profissionais, como bioquímicos, biólogos, químicos, biomédicos, estiveram presentes em menos de $25 \%$ das unidades (Tabela 1).

Os laboratórios respondentes informaram ter em seus quadros técnicos 900 médicos citopatologistas, 817 anatomopatologistas, 707 bioquímicos, 333 biomédicos, 211 biólogos, 141 patologistas clínicos e 20 químicos. Além desses profissionais de nível superior, 993 citotécnicos participaram do processo de leitura das lâminas (dados não mostrados).

A Tabela 2 apresenta o número de laboratórios por UF e sua distribuição quanto à:

- presença de citopatologistas ou patologistas na equipe;

- natureza da organização (se privada ou pública);
- forma de gestão (se municipal, estadual ou federal);

- descrição do tipo de unidade (se laboratório independente ou pertencente a uma unidade hospitalar ou ambulatorial);

- realização de exame histopatológico;

- leitura de 15 mil exames ou mais por ano;

- presença de citotécnicos.

\section{Análise dos resultados dos exames citopatoló- gicos ( $n=1.088$ laboratórios)}

Em 2002 foram realizados no Brasil 11.881.515 exames citopatológicos cervicovaginais (procedimento 12.011.01) pelo SUS. Desses, o DATASUS disponibilizou em sua página na internet informações referentes a 10.505 .773 exames $(88,4 \%)$. A distribuição dos resultados conforme o grau de qualidade da amostra e lesões detectadas é apresentada na Tabela 3. Observou-se ainda que o percentual de amostras insatisfatórias foi de 1,66\%, enquanto as consideradas com sua satisfatoriedade limitada por alguma razão corresponderam a $4,97 \%$. Já o percentual de amostras satisfatórias foi de $93,37 \%$.

Em relação às causas de não-satisfatoriedade da amostra, observou-se que, das 114.415 amostras consideradas insatisfatórias, 36,4\% o foram por dessecamento do esfregaço; $26,5 \%$ por esfregaço escasso ou hemorrágico; $9,7 \%$ por identificação incorreta da lâmina e/ou lâmina danificada; 9,3\% por esfregaço purulento; $3,3 \%$ por esfregaço com áreas espessas; e 14,8\% por outras causas (dados não mostrados).

As alterações em células escamosas mais freqüentemente observadas foram ASCUS e HPV. As NICs (graus I, II e III) foram observadas, respectivamente, em 0,84\%, 0,20\% e $0,17 \%$ dos exames. A identificação de lesão compatível com carcinoma escamoso invasivo ocorreu em $0,05 \%$ dos exames. No que diz respeito às alterações em células glandulares, AGUS foram identificadas em 0,14\% dos exames, enquanto lesões compatíveis com adenocarcinoma in situ e adenocarcinoma invasivo foram descritas, cada uma, em 0,01\% dos casos (Tabela 3).

Também se observou que em 480 laboratórios $(44,1 \%)$ nenhum caso de carcinoma escamoso foi identificado ao longo do ano. Os adenocarcinomas in situ e o invasor, diagnósticos mais raros, somente foram observados em $24,6 \%$ (268) e $21,5 \%$ (234) dos laboratórios, respectivamente (dados não mostrados).

O percentual de laboratórios que apresentou ASCUS $\geq 1,70 \%$, AGUS $\geq 0,30 \%$, razão ASCUS/ACE $\geq 0,876 \mathrm{e}$ 
Características dos laboratórios de citopatologia prestadores de serviço ao SUS ( $n=739$ ), conforme 0 número anual de exames processados, a natureza da organização, 0 tipo de gestão, o tipo de unidade, a realização de exames histopatológicos e as categorias profissionais que

Tabela 1 neles trabalham (MS, 2002)

Características

Número de laboratórios

$\%$

- Laboratórios de citopatologia prestadores de serviço ao SUS

Responderam ao estudo

Não responderam ao estudo

- Número de exames realizados no ano

$<15$ mil lâminas

$\geq 15$ mil lâminas

- Natureza da organização

Privada

Pública

Fundação beneficente ou ONG

Não informada

- Tipo de gestão

Municipal

Estadual

Não informada

- Tipo de unidade

Laboratório isolado

Laboratório vinculado a uma unidade hospitalar ou ambulatorial

- Realização de exame histopatológico

Sim

Não

Ausência de informação

- Categoria profissional do responsável técnico pelo laboratório

Médico anatomopatologista

Médico citopatologista

Bioquímico

Médico patologista clínico

Biomédico

Médico de outra especialidade

Biólogo

Farmacêutico

Químico

Ausência de informação

- Número de profissionais de nível superior envolvidos no processamento dos exames*

Médicos citopatologistas 
$\rightarrow$

Bioquímicos

273

36,9

Biólogos

128

17,3

Biomédicos

127

17,2

Médico patologista clínico

102

13,8

Químicos

\section{- Profissionais de nível médio}

Fonte: Dados primários.

Categorias não-excludentes; os percentuais referem-se à presença dessas categorias profissionais nos laboratórios e, portanto, sua soma ultrapassa 100\%.

SUS: Sistema Único de Saúde; ONG: organização não-governamental, MS: Ministério da Saúde.

\section{Distribuição dos laboratórios prestadores de serviço ao SUS por unidade da federação (UF), conforme o percentual* de participação no estudo, o número anual de exames processados, a natureza da organização, os tipos de gestão e de unidade, a realizaçäo de exames \\ Tabela 2 histopatológicos e as categorias profissionais que neles trabalham (MS, 2002)}

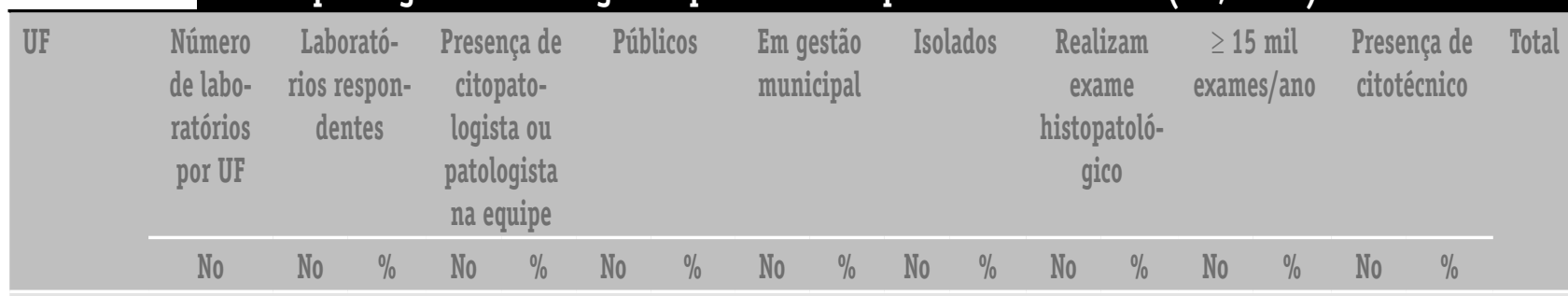

\section{- Regiäo Norte}

\begin{tabular}{|c|c|c|c|c|c|c|c|c|c|c|c|c|c|c|c|c|c|c|}
\hline Acre & 2 & 2 & $(100)$ & 1 & $(50)$ & 2 & $(100)$ & 0 & (0) & 0 & $(0)$ & 1 & (50) & 1 & (50) & 2 & $(100)$ & 2 \\
\hline Amapá & 1 & 1 & $(100)$ & 1 & $(100)$ & - & $(-)$ & 0 & (0) & 0 & (0) & 0 & (0) & 1 & $(100)$ & 1 & $(100)$ & 1 \\
\hline Amazonas & 17 & 13 & $(76,5)$ & 7 & $(53,8)$ & 4 & $(30,8)$ & 0 & (0) & 8 & $(61,5)$ & 7 & $(58,3)$ & 2 & $(15,4)$ & 9 & $(69,2)$ & 13 \\
\hline Pará** & $60^{*}$ & 0 & (0) & 0 & (0) & - & $(-)$ & - & $(-)$ & - & - & - & $(-)$ & - & $(-)$ & - & $(-)$ & - \\
\hline Rondônia & 15 & 13 & $(86,7)$ & 2 & $(15,4)$ & 0 & (0) & 1 & $(12,5)$ & 12 & $(92,3)$ & 11 & $(84,6)$ & 0 & $(0)$ & 6 & $(46,2)$ & 13 \\
\hline Roraima & 3 & 1 & $(33,3)$ & 1 & $(100)$ & 0 & (0) & $\cdot$ & $(-)$ & 0 & $(0)$ & 1 & $(100)$ & 0 & (0) & 1 & $(100)$ & 1 \\
\hline Tocantins & 10 & 8 & $(80)$ & 7 & $(87,5)$ & 0 & (0) & 5 & $(71,4)$ & 6 & (75) & 3 & $(37,5)$ & 1 & $(12,5)$ & 3 & $(37,5)$ & 8 \\
\hline \multicolumn{19}{|c|}{ - Regiäo Nordeste } \\
\hline Alagoas & 32 & 19 & $(59,4)$ & 17 & $(89,5)$ & 3 & $(15,8)$ & 13 & $(86,7)$ & 17 & $(89,5)$ & 5 & $(26,3)$ & 4 & $(21,1)$ & 10 & $(52,6)$ & 19 \\
\hline Bahia & 134 & 100 & $(74,6)$ & 69 & $(69)$ & 13 & $(13,7)$ & 43 & $(51,8)$ & 46 & $(46)$ & 77 & $(80,2)$ & 3 & (3) & 30 & $(30)$ & 100 \\
\hline Ceará & 41 & 37 & $(90,2)$ & 32 & $(86,5)$ & 7 & (20) & 28 & $(87,5)$ & 15 & $(40,5)$ & 25 & $(75,8)$ & 12 & $(32,4)$ & 23 & $(62,2)$ & 37 \\
\hline Maranhão & 37 & 23 & $(62,2)$ & 13 & $(56,5)$ & 3 & $(13)$ & 9 & $(47,4)$ & 15 & $(65,2)$ & 12 & $(57,1)$ & 7 & $(30,4)$ & 9 & $(39,1)$ & 23 \\
\hline Paraiba & 24 & 18 & (75) & 14 & $(77,8)$ & 3 & $(18,8)$ & 13 & $(86,7)$ & 13 & $(72,2)$ & 7 & $(38,9)$ & 5 & $(27,8)$ & 10 & $(55,6)$ & 18 \\
\hline Pernambuco & 64 & 44 & $(68,8)$ & 38 & $(86,4)$ & 21 & $(52,5)$ & 20 & $(71,4)$ & 17 & $(38,6)$ & 30 & $(73,2)$ & 3 & $(6,8)$ & 32 & $(72,7)$ & 44 \\
\hline Piaúi & 35 & 30 & $(85,7)$ & 30 & (100) & 1 & $(3,4)$ & 12 & (50) & 20 & $(66,7)$ & 22 & $(75,9)$ & 3 & $(10)$ & 23 & $(76,7)$ & 30 \\
\hline $\begin{array}{l}\text { Rio Grande } \\
\text { do Norte }\end{array}$ & 20 & 16 & $(80)$ & 15 & $(93,8)$ & 2 & $(13,3)$ & 8 & $(61,5)$ & 10 & $(62,5)$ & 11 & $(68,8)$ & 6 & $(37,5)$ & 16 & $(100)$ & 16 \\
\hline Sergipe & 20 & 11 & (55) & 11 & $(100)$ & 0 & (0) & 6 & $(66,7)$ & 7 & $(63,6)$ & 10 & $(90,9)$ & 1 & $(9,1)$ & 5 & $(45,5)$ & 11 \\
\hline \multicolumn{19}{|c|}{ - Regiäo Centro-oeste } \\
\hline $\begin{array}{l}\text { Distrito } \\
\text { Federal }\end{array}$ & 12 & 0 & (0) & 0 & (0) & - & $(-)$ & - & $(-)$ & & $(-)$ & - & $(-)$ & - & $(-)$ & - & $(-)$ & - \\
\hline Goiás & 55 & 37 & $(67,3)$ & 27 & (73) & 2 & $(5,6)$ & 24 & (96) & 31 & $(83,8)$ & 11 & $(29,7)$ & 2 & $(5,4)$ & 21 & $(56,8)$ & 37 \\
\hline
\end{tabular}




\begin{tabular}{|c|c|c|c|c|c|c|c|c|c|c|c|c|c|c|c|c|c|c|}
\hline $\begin{array}{l}\text { Mato Grosso } \\
\text { do Sul }\end{array}$ & 8 & 6 & (75) & 6 & $(100)$ & 0 & (0) & 6 & $(100)$ & 6 & $(100)$ & 0 & (0) & 4 & $(66,7)$ & 5 & $(83,3)$ & 6 \\
\hline $\begin{array}{l}\text { Mato Grosso } \\
\text { - Regiäo Sudest }\end{array}$ & 21 & 16 & $(76,2)$ & 9 & $(56,3)$ & 2 & $(12,5)$ & 4 & $(36,4)$ & 12 & (75) & 8 & (50) & 1 & $(6,3)$ & 5 & $(31,3)$ & 16 \\
\hline Espirito Santo & 29 & 16 & $(55,2)$ & 12 & $(75)$ & 0 & (0) & 11 & $(91,7)$ & 12 & (75) & 5 & $(31,3)$ & 5 & $(31,3)$ & 11 & $(68,8)$ & 16 \\
\hline Minas Gerais & 117 & 95 & $(81,2)$ & 66 & $(69,5)$ & 14 & $(15,4)$ & 57 & $(83,8)$ & 62 & $(65,3)$ & 42 & $(45,2)$ & 23 & $(24,2)$ & 52 & $(54,7)$ & 95 \\
\hline $\begin{array}{l}\text { Rio de } \\
\text { Janeiro }\end{array}$ & 82 & 57 & $(69,5)$ & 51 & $(89,5)$ & 14 & $(25,9)$ & 32 & $(88,9)$ & 32 & $(56,1)$ & 13 & $(22,8)$ & 4 & (7) & 31 & $(54,4)$ & 57 \\
\hline $\begin{array}{l}\text { São Paulo } \\
\text { - Regiäo Sul }\end{array}$ & 139 & 87 & $(62,6)$ & 68 & $(78,2)$ & 33 & $(41,8)$ & 41 & $(61,2)$ & 34 & $(39,1)$ & 27 & $(31,4)$ & 24 & $(27,6)$ & 53 & $(60,9)$ & 87 \\
\hline Paraná & 13 & 10 & $(76,9)$ & 10 & $(100)$ & 0 & (0) & 3 & $(60)$ & 8 & (80) & 0 & (0) & 10 & $(100)$ & 9 & $(90)$ & 10 \\
\hline $\begin{array}{l}\text { Rio Grande } \\
\text { do Sul }\end{array}$ & 57 & 45 & $(78,9)$ & 40 & $(88,9)$ & 2 & $(4,8)$ & 16 & $(59,3)$ & 35 & $(77,8)$ & 18 & $(40,9)$ & 10 & $(22,2)$ & 22 & $(48,9)$ & 45 \\
\hline $\begin{array}{l}\text { Santa } \\
\text { Catarina }\end{array}$ & 40 & 34 & $(85)$ & 26 & $(76,5)$ & 6 & $(17,6)$ & 19 & $(70,4)$ & 26 & $(76,5)$ & 14 & $(42,4)$ & 8 & $(23,5)$ & 22 & $(64,7)$ & 34 \\
\hline Total & 1.088 & 739 & $(67,9)$ & 573 & $(77,5)$ & 132 & $(18,8)$ & 371 & $(67,8)$ & 444 & $(60,1)$ & 360 & $(50,1)$ & 140 & $(18,9)$ & 411 & $(55,6)$ & 739 \\
\hline
\end{tabular}

\section{Distribuição dos resultados conforme 0 grau de satisfatoriedade da amostra e alteraçōes}

Tabela 3 identificadas (MS, 2002)

\begin{tabular}{lcc}
\hline Resultados & Número \\
• Classificação quanto à satisfatoriedade da amostra & & \\
Satisfatória & 6.431 .436 & 93,37 \\
Limitada & 342.546 & 4,97 \\
Insatisfatória & 114.415 & 1,66 \\
•Alterações em células escamosas* & & \\
ASCUS & 87.121 & 1,26 \\
HPV & 64.044 & 0,93 \\
NIC I & 57.986 & 0,84 \\
NIC II & 13.796 & 0,20 \\
NIC III & 11.730 & 0,17 \\
Carcinoma escamoso invasivo & 3.550 & 0,05 \\
•Alterações em células glandulares* & & \\
AGUS & 9.578 & 0,14 \\
Adenocarcinoma in situ & 701 & 0,01 \\
Adenocarcinoma invasivo & 554 & 0,01 \\
Total de exames & 10.505 .773 & 100 \\
\hline
\end{tabular}

Fonte: dados primários.

* Percentuais em relação ao número total de exames com informação.

MS: Ministério da Saúde; ASCUS: células escamosas atípicas de significado indeterminado; HPV: efeito citopático compatível com infecção pelo HPV; NIC I: neoplasia intraepitelial cervical grau I; NIC II: neoplasia intra-epitelial cervical grau II; NIC III: neoplasia intra-epitelial cervical grau III; ACUS: células glandulares atípicas de significado indeterminado. 
amostras insatisfatórias $\geq 2,07 \%$, que equivalem ao percentil 75 dessas freqüências, é apresentado na Tabela 4 para cada UF.

A Tabela 5 reproduz com dados nacionais as estatísticas que devem ser apresentadas pelos laboratórios norte-americanos no relatório estatístico anual de citopatologia, conforme proposta do $\mathrm{CAP}^{(6)}$. Para a construção dessa tabela, as taxas referentes a cada diagnóstico foram ordenadas da menor para a maior, sendo o percentil 50 equivalente ao valor mediano. Assim, ao observarmos os números apresentados na primeira linha da tabela, identificamos que metade dos laboratórios (percentil 50) apresentou menos de 0,76\% de resultados com ASCUS. Por outro lado, para 95\% dos laboratórios (percentil 95) a taxa de ASCUS foi $<4,58 \%$. Já para as lesões de baixo grau, os dados da segunda linha da Tabela 5 mostram que em $25 \%$ dos laboratórios o percentual foi menor que 0,58 enquanto em outros $25 \%$ os valores foram superiores a 2,61\%. Os resultados são apresentados ainda para lesões de alto grau, ACE, razão ASCUS/ACE, AGUS e amostras insatisfatórias.

\section{Fatores associados a percentuais elevados de ASCUS, AGUS, razão ASCUS/ACE e amostras insatisfatórias}

A Tabela 6 apresenta a significância estatística da associação entre algumas características selecionadas dos

Distribuição proporcional dos laboratórios prestadores de serviço ao SUS, por Unidade da

Federação (UF), conforme os percentuais de alterações selecionadas e de insatisfatoriedade da

Tabela 4 amostra acima dos limites estabelecidos (percentil 75 da distribuição percentual)* (MS, 2002)

\begin{tabular}{|c|c|c|c|c|c|c|c|c|c|}
\hline \multirow[t]{2}{*}{ Estado } & \multicolumn{2}{|c|}{$\begin{array}{c}\text { ASCUS } \\
\geq 1,70 \%\end{array}$} & \multicolumn{2}{|c|}{$\begin{array}{c}\text { AGUS } \\
\geq 0,30 \%\end{array}$} & \multicolumn{2}{|c|}{$\begin{array}{c}\text { Razão ASCUS/ACE } \\
\geq 0,876\end{array}$} & \multicolumn{2}{|c|}{$\begin{array}{l}\text { Amostra insatisfatória } \\
\qquad 2,07 \%\end{array}$} & \multirow[t]{2}{*}{ Total } \\
\hline & No & $\%$ & No & $\%$ & No & $\%$ & No & $\%$ & \\
\hline \multicolumn{10}{|l|}{ - Região Norte } \\
\hline Acre & 2 & $(100)$ & 1 & $(50)$ & 1 & (50) & 1 & (50) & 2 \\
\hline Amapá & 0 & (0) & 0 & $(0)$ & 0 & (0) & 1 & $(100)$ & 1 \\
\hline Amazonas & 7 & $(41,2)$ & 6 & $(35,3)$ & 1 & $(5,9)$ & 17 & $(100)$ & 17 \\
\hline Pará & 23 & $(38,3)$ & 31 & $(51,7)$ & 16 & $(26,7)$ & 22 & $(36,7)$ & 60 \\
\hline Rondônia & 2 & $(13,3)$ & 2 & $(13,3)$ & 3 & (20) & 3 & (20) & 15 \\
\hline Roraima & 0 & (0) & 2 & $(66,7)$ & 0 & (0) & 0 & (0) & 3 \\
\hline Tocantins & 4 & (40) & 5 & $(50)$ & 1 & (10) & 6 & (60) & 10 \\
\hline \multicolumn{10}{|l|}{ - Região Nordeste } \\
\hline Alagoas & 6 & $(18,8)$ & 5 & $(15,6)$ & 12 & $(37,5)$ & 10 & $(31,3)$ & 32 \\
\hline Bahia & 26 & $(19,4)$ & 45 & $(33,6)$ & 22 & $(16,4)$ & 36 & $(26,9)$ & 134 \\
\hline Ceará & 17 & $(41,5)$ & 10 & $(24,4)$ & 14 & $(34,1)$ & 20 & $(48,8)$ & 41 \\
\hline Maranhão & 10 & (27) & 13 & $(35,1)$ & 8 & $(21,6)$ & 15 & $(40,5)$ & 37 \\
\hline Paraíba & 7 & $(29,2)$ & 5 & $(20,8)$ & 7 & $(29,2)$ & 6 & (25) & 24 \\
\hline Pernambuco & 4 & $(6,3)$ & 7 & $(10,9)$ & 4 & $(6,3)$ & 35 & $(54,7)$ & 64 \\
\hline Piauí & 6 & $(17,1)$ & 10 & $(28,6)$ & 4 & $(11,4)$ & 1 & $(2,9)$ & 35 \\
\hline Rio Grande do Norte & 8 & (40) & 5 & $(25)$ & 3 & (15) & 16 & (80) & 20 \\
\hline Sergipe & 2 & (10) & 2 & $(10)$ & 5 & (25) & 3 & (15) & 20 \\
\hline \multicolumn{10}{|c|}{ - Região Centro-oeste } \\
\hline Distrito Federal & 1 & $(8,3)$ & 1 & $(8,3)$ & 3 & (25) & 2 & $(16,7)$ & 12 \\
\hline Goiás & 12 & $(21,8)$ & 8 & $(14,5)$ & 16 & $(29,1)$ & 5 & $(9,1)$ & 55 \\
\hline Mato Grosso do Sul & 5 & $(62,5)$ & 4 & $(50)$ & 2 & (25) & 1 & $(12,5)$ & 8 \\
\hline Mato Grosso & 7 & $(33,3)$ & 7 & $(33,3)$ & 4 & (19) & 2 & $(9,5)$ & 21 \\
\hline \multicolumn{10}{|l|}{ - Região Sudeste } \\
\hline Espírito Santo & 7 & $(24,1)$ & 5 & $(17,2)$ & 7 & $(24,1)$ & 5 & $(17,2)$ & 29 \\
\hline
\end{tabular}




\begin{tabular}{lccccccccc} 
Minas Gerais & 22 & $(18,8)$ & 15 & $(12,8)$ & 16 & $(13,7)$ & 16 & $(13,7)$ & 117 \\
Rio de Janeiro & 29 & $(35,4)$ & 24 & $(29,3)$ & 32 & $(39)$ & 14 & $(17,1)$ & 82 \\
São Paulo & 24 & $(17,3)$ & 19 & $(13,7)$ & 32 & $(23)$ & 26 & $(18,7)$ & 139 \\
- Região Sul & & & & & & & & & \\
Paraná & 0 & $(0)$ & 0 & $(0)$ & 13 & $(100)$ & 1 & $(7,7)$ & 13 \\
Rio Grande do Sul & 19 & $(33,3)$ & 14 & $(24,6)$ & 14 & $(24,6)$ & 4 & $(7)$ & 57 \\
Santa Catarina & 14 & $(35)$ & 12 & $(30)$ & 17 & $(42,5)$ & 4 & $(10)$ & 40 \\
Total & 264 & $(24,3)$ & 258 & $(23,7)$ & 257 & $(23,6)$ & 272 & $(25)$ & 1.088 \\
\hline
\end{tabular}

Fontes: Departamento de Informática do Sistema Único de Saúde (DATASUS), 2004 e dados primários.

Percentuais em relação ao número de laboratórios do estado.

0 percentil 75 foi definido como valor crítico para estabelecimento dos pontos de corte.

MS: Ministério da Saúde.

\section{Tabela 5 Percentuais de alterações citopatológicas conforme o percentil selecionado (MS, 2002)}

\begin{tabular}{lccccccc} 
Categoria & \multicolumn{7}{c}{ Percentis das taxas } \\
& $5^{\circ}$ & $10^{\circ}$ & $25^{\circ}$ & $50^{\circ}$ & $75^{\circ}$ & $90^{\circ}$ & $95^{\circ}$ \\
ASCUS (\%*) & 0 & 0,08 & 0,31 & 0,76 & 1,67 & 3,24 & 4,58 \\
Lesão de baixo grau $\left(\%{ }^{*}\right)$ & 0 & 0,15 & 0,58 & 1,35 & 2,61 & 4,74 & 7,09 \\
Lesão de alto grau $\left(\%{ }^{*}\right)$ & 0 & 0,06 & 0,37 & 0,90 & 1,71 & 3,15 & 4,99 \\
Alterações em células escamosas $\left(\%{ }^{*}\right)$ & 0 & 0,29 & 0,88 & 1,83 & 3,36 & 6,10 & 8,96 \\
Razão ASCUS/ACE & 0,02 & 0,08 & 0,20 & 0,46 & 0,88 & 1,54 & 2,26 \\
AGUS (\%*) & 0 & 0 & 0 & 0,09 & 0,28 & 0,67 & 1,20 \\
Amostra insatisfatória $(\% *$ & 0 & 0 & 0,23 & 0,73 & 2,07 & 5,32 & 8,30 \\
\hline
\end{tabular}

Fonte: Dados primários.

* Percentuais em relação ao número total de exames com informação.

ASCUS: células escamosas atípicas de significado indeterminado; lesão de baixo grau: efeito citopático compatível com infecção pelo papilomavírus humano (HPV) + neoplasia intra-epitelial cervical grau I (NIC I); lesão de alto grau: neoplasia intra-epitelial cervical grau II (NIC II) + neoplasia intra-epitelial cervical grau III (NIC III); ACUS: células glandulares atípicas de significado indeterminado; ACE: alterações em células escamosas (HPV + NIC I + NIC II + NIC III + carcinoma escamoso invasor).

\section{Tabela 6}

Significância estatística (valor de $\mathrm{p}$ ) * para associação entre características dos laboratórios e

\begin{tabular}{lcccc}
\hline Características & ASCUS $\geq$ & AGUS $\geq$ & Razão ASCUS/ACE & Insatisfatório $\geq$ \\
& $1,70 \%$ & $0,30 \%$ & $\geq 0,876$ & $2,07 \%$ \\
$\begin{array}{l}\text { Ausência de médico patologista ou } \\
\text { citopatologista na equipe }\end{array}$ & 0,38 & $<0,001$ & 0,05 & 0,27 \\
Organização privada, beneficente e/ou 0NG & 0,65 & 0,99 & 0,08 & 0,049 \\
Gestão municipal & 0,43 & 0,31 & 0,84 & 0,09 \\
Laboratório vinculado a outra unidade de & 0,04 & 0,10 & 0,24 & 0,06 \\
saúde & & & & \\
Não-realização de exame histopatológico & 0,54 & 0,28 & 0,0002 & $0,006 * *$ \\
Realização de menos de 15 mil exames/ano & 0,24 & $<0,001$ & 0,54 & 0,60 \\
Presença de citotécnico no laboratório & 0,57 & 0,21 & 0,54 & 0,36 \\
\hline
\end{tabular}

Fontes: DATASUS e dados primários.

* Teste do qui-quadrado ( $\chi 2)$ bilateral; as diferenças estatisticamente significativas $(p<0,05)$ estão destacadas em negrito.

${ }^{* *}$ Associação positiva entre percentual de exames insatisfatórios $\geq 2,07$ com a realização de exame histopatológico.

ASCUS: células escamosas atípicas de significado indeterminado; ACUS: células glandulares atípicas de significado indeterminado; ACE: alterações em células escamosas (HPV

+ NIC I + NIC II + NIC III + carcinoma escamoso invasor); DATASUS: Departamento de Informática do Sistema Único de Saúde; ONG: organização não-governamental. 
laboratórios e percentuais elevados de ASCUS, de AGUS, de amostras insatisfatórias e da razão ASCUS/ACE (considerando como valores críticos os que são superiores ao percentil 75 da sua distribuição). Os resultados apontam que a vinculação do laboratório a uma outra unidade de saúde mostrou associação estatisticamente significativa com índices elevados de ASCUS. Além disso, não ter em seu quadro médicos patologistas e/ou citopatologistas e realizar menos de 15 mil exames/ano estiveram associados a aumento na probabilidade de apresentar índices elevados de AGUS. Por outro lado, não realizar exames histopatológicos esteve associado a aumento na probabilidade de apresentar valores elevados para a razão ASCUS/ACE. Inversamente, a realização de exames histopatológicos pelos laboratórios mostrou-se associada a elevado percentual de exames insatisfatórios, bem como o fato de ser organização privada, beneficente e/ou ONG.

\section{Discussão e comentários}

No Brasil, as preocupações com a qualidade dos exames têm acompanhado a implementação das ações de detecção precoce do câncer do colo do útero. Como iniciativas nacionais nessa área, devem-se destacar o Seminário $\mathrm{Na}$ cional sobre Nomenclatura e Controle de Qualidade dos Exames Citológicos e Prevenção do Câncer Cérvico-uterino, ocorrido em 1993(14), assim como as oficinas de trabalho que definiram a nomenclatura brasileira para laudos citopatológicos cervicais e condutas clínicas preconizadas, e as recomendações para o monitoramento externo da qualidade dos exames citopatológicos ${ }^{(15,19)}$.

Junto a essas ações, a criação de um aplicativo para a informatização dos resultados dos exames citopatológicos, - SISCOLO, além de se disponibilizar uma síntese estatística das principais informações dele provenientes na página do DATASUS na internet, representam importantes instrumentos para monitoramento e avaliação das ações dirigidas ao controle do câncer do colo do útero no Brasil.

Os dados de identificação da mulher, informações demográficas e epidemiológicas e o laudo padronizado do exame citopatológico são registrados no SISCOLO, mas o pagamento dos exames processados deve ser condicionado ao repasse dessas informações pelo laboratório prestador de serviço ao município gestor, conforme definido em portaria do $\mathrm{MS}^{(20)}$.

Os resultados obtidos em nosso estudo mostraram que, dos 11,9 milhões de exames citopatológicos apresentados ao SIA/SUS para pagamento em $2002,88,4 \%$ tiveram seus resultados digitados no SISCOLO e disponibilizados pelo DATASUS na internet. Dos 1.028 laboratórios convidados a participar deste estudo, 739 (71,9\%) responderam à enquete versando sobre o perfil dessas unidades, o que pode se considerar uma elevada taxa de resposta.

Quanto ao perfil desses laboratórios, observa-se grande variabilidade no número de laboratórios por UF (1 a 139). 0 mesmo fato se repete no que se refere à classificação desses laboratórios em relação ao número anual de exames processados, à natureza da organização, aos tipos de gestão e de unidade, à realização de exames histopatológicos e às categorias dos profissionais envolvidos na leitura dos exames.

Merecem destaque os seguintes aspectos: o fato de que a maior parte dos laboratórios $(81,1 \%)$ processa um número de exames inferior ao recomendado como mínimo ideal (15 mil/ano) pela OPS(21); a natureza privada da maioria dos laboratórios (70,2\%); a condição de serem desvinculados de unidade hospitalar ou ambulatorial $(60,1 \%)$; e ter médicos como responsáveis técnicos (74,8\%). Devemos considerar que no SISCOLO constam apenas os exames realizados para o SUS e, conseqüentemente, é esperado que um percentual maior de laboratórios privados deva fazer mais de 15 mil exames por ano, ainda que esses dados não estejam disponíveis.

Paralelamente, os valores medianos para lesões de baixo grau identificados neste estudo são semelhantes àqueles observados (em 2003) nos EUA $(1,4 \%)^{(6)}$, enquanto as medianas para os percentuais de ASCUS, AGUS e para a razão ASCUS/ACE mostraram-se inferiores aos valores norte-americanos $(3,2 \%, 0,2 \% \text { e } 1,4 \% \text {, respectivamente })^{(6)}$. Somente no caso do percentual mediano de exames com lesão de alto grau foram observados valores mais elevados nos dados nacionais, quando em comparação com os dos EUA $(0,4 \%)^{(16)}$. Esse fato sugere que, além dos problemas relacionados à baixa concordância entre observadores para alguns diagnósticos ${ }^{(11,24)}$, pode ser que o sistema de Bethesda não esteja sendo adequadamente usado ou que esses resultados sejam reflexo das práticas mais defensivas adotadas nos EUA. Portanto, considerando que a taxa de incidência padronizada do câncer do colo do útero para 2002 nos EUA ${ }^{(10)}$ é quase três vezes menor $(7,7$ por 100 mil mulheres) do que a estimada pelo INCA ${ }^{(16)}$ para o Brasil (20,31 por 100 mil mulheres), os resultados por nós obtidos para lesões de alto grau podem indicar, comparativamente, o elevado risco para as mulheres que estão sendo rastreadas.

De qualquer modo, deve-se ainda considerar a forma oportunista de rastreamento desenvolvida no país, ou 
seja, a realização dos exames por demanda espontânea, permitindo a inclusão de mulheres em faixas etárias mais jovens e com pequeno intervalo entre os exames, grupo que sabidamente apresenta baixo risco para apresentar câncer do colo uterino(9). Esse dado é reforçado pela observação de que, nos dois anos anteriores à pesquisa (2000 e 2001), $67,5 \%$ dos exames foram realizados em mulheres fora da faixa etária prioritária do Programa Nacional de Prevenção do Câncer do Colo Uterino (PNPCCU), 35 a 49 anos, e somente $15,3 \%$ e $13,7 \%$, respectivamente, estavam sendo submetidas ao primeiro exame na vida ${ }^{(27)}$. Já em 2002, em virtude da intensificação de ações nacionais por meio de uma campanha, o percentual de exames fora da faixa etária prioritária foi de $42,1 \%$ e o percentual de mulheres que estavam sendo submetidas ao seu primeiro exame na vida foi de $20,2 \%{ }^{(15)}$, o que permite especular que a situação no país seja ainda mais grave.

Segundo o $\mathrm{CAP}^{(6)}$, a razão ASCUS/ACE é útil para comparações interlaboratoriais, visto que o número de lesões em células escamosas varia enormemente entre laboratórios. Essa diferença pode ser parcialmente devido à variação geográfica da prevalência das lesões e/ou à variabilidade de critérios diagnósticos entre os laboratórios ${ }^{(7)}$.

Observa-se, por exemplo, que laboratórios que atendem à prática privada tendem a apresentar menor freqüência de infecções pelo HPV, o que deveria ser acompanhado por menor freqüência de $\mathrm{ACE}$, fazendo com que essa razão possa ser utilizada para detectar a sub ou superinterpretação das ASCUS(6). Por exemplo, em um laboratório com taxa de ASCUS superior a 3\% parece que há hiperdiagnóstico, uma vez que esse valor é maior que o percentil 75 para a distribuição nacional desse diagnóstico. Entretanto, se esse mesmo laboratório tem um percentual de alterações em células escamosas de $6 \%$, a razão ASCUS/ACE será de 0,50 , o que está próximo da mediana nacional. Logo se pode concluir que esse laboratório atende a uma população de alto risco para câncer do colo uterino. Diferentemente, um laboratório com 1\% de ASCUS e 0,25\% de ACE, embora apresente taxas de ASCUS próximas ao valor mediano, ele tem uma razão ASCUS/ACE de 4, que é muito maior do que a encontrada na maior parte dos laboratórios (95\% dos laboratórios apresentaram razão ASCUS/ACE < 2,26).

Algumas características dos laboratórios estiveram associadas a percentuais elevados de ASCUS, AGUS, razão ASCUS/ACE e ao percentual de amostras insatisfatórias. Evidentemente, esses dados devem ser analisados com a ressalva de que pode haver diferenças importantes nas características dos exames encaminhados a cada laboratório. Além disso, alguns laboratórios podem receber proporcionalmente mais exames oriundos do rastreamento do câncer do colo uterino, enquanto outros podem estar vinculados a ambulatórios de patologia cervical, em que a concentração de exames alterados é maior, principalmente com diagnósticos de maior gravidade. Esse fato explicaria, em parte, a variabilidade para todos os diagnósticos citopatológicos observada entre os laboratórios.

Um provável limite deste estudo se refere ao desconhecimento do perfil dos laboratórios não-participantes $(28,1 \%)$ e daqueles não-vinculados ao SUS. Uma análise das informações disponíveis sobre os resultados dos exames citopatológicos mostrou não haver diferença estatisticamente significativa $(p>0,05)$ no número médio de exames processados no ano, bem como nos percentuais medianos de resultados insatisfatórios, ASCUS e AGUS entre os laboratórios participantes $(n=739)$ e aqueles que não responderam à enquete $(n=349)$. Todavia, a atribuição da qualidade do esfregaço depende muito do rigor com que se avalia. Assim, a garantia interna de qualidade tende a caracterizar exames previamente considerados adequados como insatisfatórios ou como de satisfatoriedade limitada ${ }^{(2)}$.

Por fim, de maneira semelhante ao $\mathrm{CAP}^{(6)}$, propomos que os laboratórios brasileiros tenham os principais indicadores de desempenho monitorados e que aqueles com razão anual ASCUS $/ \mathrm{ACE}<5^{\circ}$ ou $>95^{\circ}$ percentis documentem as razões para esses resultados, ou então que sejam alvo de controle externo rigoroso. Essa preocupação também deveria ser estendida aos laboratórios cuja prevalência dos diagnósticos apresenta grande variabilidade.

Portanto, conclui-se que uma análise mais detalhada do perfil dos laboratórios de citopatologia prestadores de serviços ao SUS é tarefa complexa, pois permite ampla inferência sobre associações, causas e conseqüências e, conseqüentemente, poderia ser objeto de um colóquio especificamente organizado com tal finalidade.

É fato que é possível avaliar a qualidade dos resultados dos exames citopatológicos a partir da análise dos dados disponibilizados pelos sistemas oficiais de informação e que o modelo apresentado já pode se constituir numa importante ferramenta para monitorá-la. Além disso, o uso rotineiro desses dados poderá fornecer subsídios para aprimorar os sistemas de informação e orientar programas de educação continuada dirigidos aos profissionais atuantes nos laboratórios e àqueles que atuam na coleta de material cervicovaginal. 


\section{Referências}

I. ABREU, E. Reorientação nas ações de prevenção e controle do câncer cérvico-uterino. Rev Bras Cancerol, v. 35, n. I/2, p. 55-8, 1989.

2. AMARAL, R.G. et al. Quality assurance of cervical smears: $100 \%$ rapid rescreening versus 10\% random rescreening. Acta Cytol, v. 49, p. 244-8, 2005.

3.AMERICAN SOCIETY OF CYTOPATHOLOGY (ASC). Cervical Cytology Practice Guidelines. Disponível em: http://www. cytopathology.org/guidelines/. Acesso em: 9 mar. 2005.

4.AQUINO, E.M.L.Avaliação da situação atual das ações de controle. Rev Bras Cancerol, v. 31, n. 3, p. I 35, 1985.

5. BABES,A. Diagnostic du cancer du col utérin par les frottis. Presse Medicale, v. 36, p. 45I-4, 1928.

6. COMMISSION ON LABORATORY ACCREDITATION. Laboratory Accreditation Program: 2005 cytopathology checklist. College of American Pathologists, 2005. Disponível em: <http://www.cap.org/apps/docs/laboratory_accreditation/ checklists/laboratory_general_april2006.pdf>. Acesso em: 10 mai. 2006.

7. CONFORTINI, M. et al. Interlaboratory reproducibility of atypical squamous cells of undetermined significance report: a national survey. Cytopathology, v. 14, p. 263-8, 2003.

8. DATASUS. Ministério da Saúde. Informações de saúde. Brasília, 2005. Disponível em: <http://www.datasus.gov.br>. Acesso em: 4 fev. 2005.

9. D'OTTAVIANO MORELLI, M.G. et al. Prevalence of cervical intraepithelial neoplasia and invasive carcinoma based on cytological screening in the region of Campinas, São Paulo, Brazil. Cad Saude Publ, v. 20, n. I53-9, 2004.

10. FERLAY, J. et al. GLOBOCAN 2002: Cancer incidence, mortality and prevalence worldwide. Lyon: IARCPress: 2004. IARC Cancer Base $n^{\circ} .5$ (version 2.0).

I I. GRENKO, R.T. et al.Variance in the interpretation of cervical biopsy specimens obtained for atypical squamous cells of undetermined significance. Am J Clin Pathol, v. I I 4, p. 735-40, 2000.

12. INSTITUTO NACIONAL DE CÂNCER (INCA). Ministério da Saúde. Inquérito Domiciliar: Comportamentos de risco e morbidade referida de agravos não transmissíveis - Brasil, I5 capitais e Distrito Federal 2002/2003. Rio de Janeiro: INCA, 2004

13. INSTITUTO NACIONAL DE CÂNCER (INCA). Ministério da Saúde: Câncer do colo do útero: informações técnico-gerenciais e ações desenvolvidas. Rio de Janeiro: INCA, 2002.

14. INSTITUTO NACIONAL DE CÂNCER (INCA). Ministério da Saúde: Seminário Nacional sobre Nomenclatura e Controle de Qualidade dos Exames Citológicos e Prevenção do Câncer Cérvico-uterino. Rio de Janeiro: INCA, 1993.

15. INSTITUTO NACIONAL DE CÂNCER (INCA). Ministério da Saúde: Nomenclatura Brasileira para Laudos Citopatológicos Cervicais e Condutas Clínicas Preconizadas. Rio de Janeiro: INCA, 2003.

16. INSTITUTO NACIONAL DE CÂNCER (INCA). Ministério da Saúde: Estimativa 2005: incidência de câncer no Brasil. Rio de Janeiro: INCA, 2004.

17. JONES, B.A.; DAVEY, D.D. Quality management in gynecologic cytology using interlaboratory comparison. Arch Pathol Lab Med, v. I24, p. 672-81, 2000.

18. LOPES, E.R. et al. Comportamento da população brasileira feminina em relação ao câncer cérvico-uterino.J bras Ginec, v. 105, n. I I/12, p. 505-16, 1995.

19. MINISTÉRIO DA SAÚDE. Nomenclatura Brasileira para Laudos Cervicais e Condutas Preconizadas. Rev Bras Canc, v. 52, n. 3, p. 2 I3-36, 2006.

20. MINISTÉRIO DA SAÚDE. Secretaria de Assistência à Saúde. Portaria SASIMS 408 de 30 de julho de 1999. Brasilia, DF: Diário Oficial da União, 1999; 2 ago.

21. ORGANIZACIÓN PANAMERICANA DE LA SALUD (OPS). Módulo de citología: procedimientos. Washington, D.C.: OPS, 2000.

22. PAPANICOLAOU, G.N. New cancer diagnosis. In: PROCEEDINGS OF THE THIRD RACE BETTERMENT CONFERENCE. Michigan: Battle Creek, 1928. Jan 2-5.

23. SIEGEL, S. Estatística não-paramétrica. Rio de Janeiro: McGraw Hill do Brasil, 1975.

24. STOLER, M.H., SCHIFFMAN, M. Interobserver reproducibility of cervical cytologic and histologic interpretations: realistic estimates from the ASCUS-LSIL Triage Study. JAMA, v. 285, p. 1500-5, 2001.

25.TAN, K.B. et al. Quality indices in a cervicovaginal cytology service: before and after laboratory accreditation. Arch Pathol Lab Med, v. 128, n. 3, p. 303-7, 2004.

26. THAMBOO,T.P. et al. Cervical cytology: an audit in a Singapore teaching hospital. Singapore Med J, v. 44, p. 256-60, 2003.

27.THULER, L.C.S. Lesões precursoras do câncer do colo uterino: aspectos epidemiológicos. In: FIGUEIREDO, E.M.A. Ginecologia oncológica. Rio de Janeiro: Revinter, 2004. p. 3-6.

28. XAVIER, F.E. História da citopatologia no Brasil. Informativo Citopatologia, 2002. Edição 59, abril a junho. Disponível em: <http://www.citopatologia.org.br>. Acesso em: 25 jan. 2005. 